\title{
1 The maize E3 ligase ZmCER9 specifically targets activated NLRs for 2 degradation
}

3 Shailesh Karre ${ }^{1}$, Saet-Byul Kim ${ }^{1}$, Devarshi Selote ${ }^{1}$, Rajdeep Khangura ${ }^{2}$, Brian Dilkes ${ }^{2}$, Guri S

4 Johal $^{3}$ and Peter Balint-Kurti ${ }^{1,4^{*}}$

5

$6 \quad{ }^{1}$ Department of Entomology and Plant Pathology, NC State University, Raleigh, USA

$7 \quad{ }^{2}$ Dept of Biochemistry, Purdue University, West Lafayette, USA

$8 \quad{ }^{3}$ Department of Botany and Plant Pathology, Purdue University, West Lafayette, USA

$9 \quad{ }^{4}$ Plant Science Research Unit USDA-ARS, NC State University, Raleigh, USA

11 The authors have withdrawn their manuscript whilst they perform additional experiments to test some of 12 their conclusions further. Therefore, the authors do not wish this work to be cited as reference for the 13 project. If you have any questions, please contact the corresponding author

14

15

16

17

18 